\title{
The Effects of Wall Populations on Coexistence of Bacteria in the Liquid Phase of Chemostat Cultures
}

\author{
By LIN CHAO* AND GISELA RAMSDELL \\ Department of Ecology and Evolutionary Biology, Northwestern University, Evanston, \\ Illinois 60201, USA
}

(Received 23 October 1984)

\begin{abstract}
We have examined the effects of wall populations on coexistence between strains of Escherichia coli in the liquid phase of mixed (two-strain) chemostats. The wall populations of the two competing strains became established soon after the start of the cultures and, although the relative abundance of the strains in the liquid phase could change over time by several orders of magnitude, the composition of an established wall population did not change markedly. The bacterial strains examined could not displace an established wall population of a competing strain. The presence of a permanent wall population allowed a strain that was less fit in the liquid phase to coexist with a superior strain. The resulting coexistence did not require that the inferior strain attached to the vessel wall better than the superior strain. We believe that the coexistence developed because the inferior strain survived and reproduced on the vessel wall. The progeny from that wall population then provided replacements for the bacteria that the inferior strain lost through a selective disadvantage in the liquid phase of the culture. By replacing the chemostat vessel, hence eliminating the wall populations, we could distinguish between cases where the coexistence depended on the presence of a wall population and where it resulted from some alternative mechanism.
\end{abstract}

\section{INTRODUCTION}

In theory, two populations of asexual organisms cannot coexist stably in a chemostat (continuous culture) limited by a single resource (Moser, 1957; Powell, 1958; Tempest, 1970; Stewart \& Levin, 1973; Taylor \& Williams, 1975; Hsu et al., 1977). In practice, such an outcome is often observed; coexistence does not develop and the inferior population is eliminated from the culture (Contois, 1959; Bungay \& Bungay, 1968; Meers \& Tempest, 1968; Meers, 1973; Jannasch \& Mateles, 1974; Chao et al., 1977). A not uncommon result, however, is that some populations eventually reach an equilibrium and coexist (Zamenhof \& Eichhorn, 1967; Edlin et al., 1975; Dykhuizen, 1978; Godwin \& Slater, 1979; Chao et al., 1983). The empirical result that two asexual strains can coexist in a chemostat culture limited by a single resource is of biological significance. It shows that, even in a habitat as simple and homogeneous as a chemostat, organismic diversity may be maintained by the existence of other limiting factors. Considering the success of studies using chemostats to support complex multispecies communities (Megee $e t$ al., 1972; Chao et al., 1977; Veilleux \& Rowland, 1981; Freter et al., 1983), it becomes important to understand the mechanisms responsible for the coexistence of organisms in such a laboratory system.

Three hypotheses are generally considered in accounting for the coexistence of bacterial strains in mixed (multistrain) chemostats. First, given two bacterial strains of unequal fitnesses, the inferior strain could generate a few beneficial mutants that are equal in fitness to the superior strain (Dykhuizen, 1978; Chao \& Cox, 1983). Though the inferior strain is eliminated, the mutants and the superior strain coexist (Powell, 1958). Second, one of the strains could grow on the metabolic by-products of the other (Powell, 1958; Shindala et al., 1965; Megee et al., 1972; 
Meers, 1973; Jannasch \& Mateles, 1974; Taylor \& Williams, 1975), in effect making the two strains limited by different resources. Finally, the inferior strain could survive by adhering to the wall of the chemostat vessel (Larsen \& Dimmick, 1964; Bungay \& Bungay, 1968; Meers, 1973; Dykhuizen, 1978; Dykhuizen \& Hartl, 1983). If the wall populations are permanent, the inferior strain cannot be eliminated from the chemostat.

Of the three hypotheses, the establishment of coexistence by the presence of a permanent wall population, or a wall effect, is the one most experimentally amenable. If a wall effect is not present, the transfer of the liquid contents of the chemostat culture to a new vessel should not disrupt the coexistence (Edlin et al., 1975). Although a few studies have used this vessel replacement test (Freter et al., 1983; Dykhuizen \& Hartl, 1983), a detailed examination of the wall effect phenomenon has not been made. This report presents the results of a study examining the effects of wall populations on the coexistence of Escherichia coli strains in the liquid phase of mixed chemostats. We investigated the coexistences using the vessel replacement test but have elaborated upon the previous studies. We sampled over time not only the liquid populations but also the wall populations in the cultures. We examined the behaviour of the liquid populations in the absence of a wall effect by replacing the chemostat vessels both before and after the coexistences were achieved. Simultaneous information on the behaviour of the liquid and the wall populations enabled us to detail more quantitatively the mechanisms responsible for a coexistence mediated by a wall effect.

\section{METHODS}

Bacterial strains. Strain P127 was E. coli K12 $\mathrm{F}^{-}$strain W3110 (Cox \& Gibson, 1974). Strains P151 (Cox \& Gibson, 1974), GR101 (this study) and CH401 (Chao et al., 1983) were all constructed from P127. Strains P151 and GR 101 carried the mutator allele $m u t T l$ and were genetically marked $\mathrm{Lac}^{-}$. Strain $\mathrm{CH} 401$ carried the transposon $\mathrm{Tn} 10$, which encodes for tetracycline resistance (Foster et al., 1981). The transposon was inserted into the lac operon, rendering the strain $\mathrm{Lac}^{-}$. Both $\mathrm{CH} 401$ and GR101, termed evolved strains, were allowed to evolve and adapt to the chemostat environment. $\mathrm{CH} 401$ was isolated in a previous study after 219 generations in a chemostat (Chao et al., 1983). GR 101 evolved from P151 and was isolated from a preliminary chemostat (results not shown) after 534 generations. Stocks were stored in $40 \%(\mathrm{v} / \mathrm{v})$ glycerol at $-20{ }^{\circ} \mathrm{C}$.

Chemostats. The chemostats had a holding volume of $40 \mathrm{ml}$ and the internal volume was regulated by a vacuumdriven overflow tube (Chao et al., 1977). A peristaltic pump (Polystaltic, Buchler Instruments, Fort Lee, NJ, USA) controlled the flow of the medium, Vogel \& Bonner (1956) minimal salts with glucose at a limiting concentration of $500 \mu \mathrm{g} \mathrm{ml}^{-1}$, at a rate of $11.4 \pm 0.50 \mathrm{ml} \mathrm{h}^{-1}$ (Mean \pm SEM, $n=15$ ). The average generation time for the chemostats, defined as $\log _{e}(2) \times$ holding volume $\div$ average flow rate (Kubitschek, 1970), was $2 \cdot 4 \mathrm{~h}$. The chemostats were established by (i) mixing overnight batch monocultures of each of the competing strains (grown in the same type of medium as that for the chemostats) at the starting ratios indicated in the figures, (ii) introducing the mixed strains into an empty chemostat as an inoculum of $40 \mathrm{ml}$ (the holding volume) without diluting the bacteria (to avoid an initial period of growth and selection at glucose concentrations much higher than that in a chemostat with mixed populations under resource limitation), and (iii) starting the aeration and flow of medium immediately. Batch and chemostat cultures were incubated at $37^{\circ} \mathrm{C}$.

All glass surfaces in the chemostat vessels were cleaned with chromic acid because the coexistence frequencies of the bacteria, when caused by a wall effect, were more reproducible between replicate experiments with acidclean glassware (preliminary studies, results not presented). Silicone-coated chemostat vessels were prepared by rinsing with a $1 \%$ (v/v) solution of Prosil-28 (PCR Research Chemicals, Inc., Gainesville, Fla., USA) and air drying for $24 \mathrm{~h}$.

Replacing chemostat vessels. Vessels were replaced by transferring with a pipette under sterile conditions the entire liquid contents of a chemostat to a clean sterile chemostat and starting the latter immediately. The vessels of the experimental chemostats were replaced periodically and the removed vessels were used for the wall samples. The vessels of the control chemostats were not replaced and a wall sample was taken only when the culture was terminated.

Sampling procedures. Samples of the liquid populations were drawn from the chemostats with a sterile Pasteur pipette (Chao \& Cox, 1983). Samples of the wall populations were obtained by first rinsing the chemostat vessels five times with $20 \mathrm{ml}$ sterile $0.8 \%(\mathrm{w} / \mathrm{v})$ saline. During each rinse the vessels were gently swirled without vortexing, to a void dislodging the wall population, and the used saline was discarded. Sterile saline $(10 \mathrm{ml})$ was then added to the vessels and vortexed vigorously for $1 \mathrm{~min}$. The resulting suspension was used as the wall sample.

The paired strains in the liquid and the wall samples were distinguished by their Lac and/or tetracycline markers by appropriately diluting the samples in saline and plating on modified tetrazolium lactose (TZL) indicator plates (Chao \& Cox, 1983), TZL plates with $20 \mu \mathrm{g}$ tetracycline $\mathrm{ml}^{-1}$, and/or Vogel \& Bonner minimal 


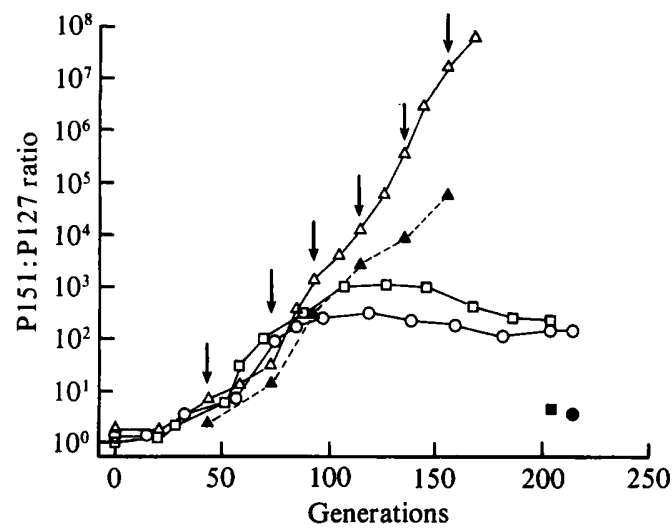

Fig. 1. Chemostats with mixed populations of P151 (mutT1 $\left.\mathrm{Lac}^{-}\right)$and P127 (mut $\left.{ }^{+} \mathrm{Lac}^{+}\right)$. Arrows denote times when the vessel of the experimental chemostat was changed. $O$, Liquid samples from control chemostat; , wall samples from control chemostat; $\Delta$, liquid samples from experimental chemostat; $\Delta$, wall samples from experimental chemostat; $\square$, liquid samples from silicone-coated chemostat; $\square$, wall samples from silicone-coated chemostat.

lactose plates. Viable counts were determined after $1 \mathrm{~d}$ at $37^{\circ} \mathrm{C}$. Dilutions were adjusted to yield approximately 300 colonies per strain per plate. White and red colonies on the TZL plates were scored respectively as $\mathrm{Lac}^{+}$and Lac $^{-}$colonies. The sensitivity of our sampling techniques was 1 bacterium $\mathrm{ml}^{-1}$. With a total density of about $5 \times 10^{8}$ bacteria $\mathrm{ml}^{-1}$ in our chemostats, the highest detectable ratio of two competing strains was about $5 \times 10^{8}$.

\section{RESULTS AND DISCUSSION}

\section{Chemostats with strains P151 and P127}

We first examined the behaviour of three control and three experimental chemostats with mixed populations of strains P151 and P127. The results of a control and an experimental chemostat, typical for this series, are presented in Fig. 1. The liquid population in the control chemostat did not change during the first 25 generations. The P151:P127 ratio then increased to about $10^{2}$ and the two populations coexisted in the liquid phase at this ratio. Although the cause of the coexistence is unknown, the initial behaviour of the liquid populations in such a $\mathrm{P} 151+\mathrm{P} 127$ chemostat is understood (Chao \& Cox, 1983). P151 is a mutator strain and it gains an advantage by generating beneficial mutants at a higher rate. The resulting advantage, however, is not detected during the first 25 generations because the mutant population, and hence its reproductive contribution, is initially too small relative to the P151 population. It is only after 25 generations that one of the beneficial mutants has become the predominant clone in the $\mathrm{P} 151$ population.

To determine if the liquid phase coexistence between P151 and P127 was caused by a wall effect, we ended the control chemostat after 214 generations and sampled the final liquid and wall populations. The final P151:P127 ratio of the wall population (3.5) was much lower than the final ratio in the liquid $\left(1.45 \times 10^{2}\right)$ and markedly closer to the starting liquid ratio of 1.2 (Fig. 1). We concluded from these results that (i) the vessel wall of the control chemostat had been colonized before the P151:P127 liquid ratio increased at the 25 generation point, and (ii) the frequency of the two strains on the wall of the same culture changed only slightly once the wall populations were established. Thus, the coexistence between P151 and P127 in the liquid phase of the control chemostat could have resulted from a wall effect. A P127 wall population would provide replacements for the bacteria that the P127 liquid population lost through a selective disadvantage. When the rate of replacement equalled the rate of loss, the two strains coexisted in the liquid phase. The coexistence would not require that P127, the less fit strain, attached to the chemostat wall better than P151. It would require only that P127 formed a permanent wall population. A permanent P151 wall population also existed in the culture, but its reproductive contribution would not have noticeably affected the coexistence because the P151 liquid population was about 100 times larger than the P127 liquid population. 


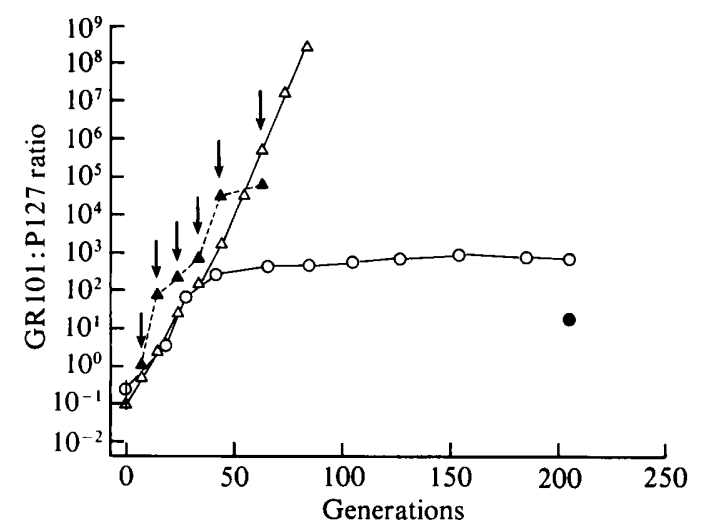

Fig. 2. Chemostats with mixed populations of GR101 (evolved, $m u t T \mathrm{Lac}^{-}$) and P127 $\left(\mathrm{mut}^{+} \mathrm{Lac}^{+}\right.$). Arrows denote times when the vessel of the experimental chemostat was changed. $O$, Liquid samples from control chemostat; $O$, wall samples from control chemostat; $\triangle$, liquid samples from experimental chemostat; $\boldsymbol{\Delta}$, wall samples from experimental chemostat.

We examined further the influence of the wall populations on the coexistence between the P127 and P151 liquid populations by replacing the vessel in the experimental chemostat. We monitored changes in the P151:P127 liquid ratio and replaced the vessel whenever the ratio increased by a factor of 10 . Although the initial behaviours of the control and the experimental chemostats were identical, the two diverged dramatically after 80 generations as the P127 liquid population in the experimental culture declined to an undetectable level (Fig. 1). The close match between the P151:P127 ratios in the first wall sample in the experimental chemostat at 43 generations and in the final wall sample in the control chemostat at 214 generations $(2.4$ and $2 \cdot 5$, respectively) supported our earlier conclusions that the wall ratio of the two strains in the control culture did not change once the wall populations were established and that the wall populations were established rapidly. Such a rapid establishment rate and the fact that in the experimental chemostat the P151:P127 ratio on the vessel wall was, at any one time, lower than that in the liquid phase led us to conclude further that (i) P127 did not attach to the vessel wall better than P151, and (ii) the bacteria in the experimental culture colonized the wall of the new vessels at a P151:P127 ratio close to that found in the liquid phase shortly after the last vessel change. The last conclusion explained why coexistence did not occur in the experimental chemostat. Since the liquid ratios in the experimental culture were increasing in favour of P151, the wall ratios increased correspondingly and the P127 wall population was slowly eliminated. Without its wall population, P127 could not coexist with P151.

To determine if silicone would prevent the strains from adhering to the chemostat wall and eliminate a wall effect, we also ran a P151 + P127 chemostat with a silicone-coated vessel. We treated this chemostat like a control culture and did not change its vessel. This coated chemostat behaved like the control culture (Fig. 1), indicating that silicone did not prevent the adhesion of bacteria, a result consistent with an earlier report (Dykhuizen, 1978).

\section{Chemostats with strains GR101 and P127}

The results described above indicated that a wall effect was contributing to the persistence of P127 in the liquid phase of the control chemostat. We surmised then that the outcome could be different if we replaced P151 with a strain that adhered to the wall of the chemostat vessel better than P127. Since bacteria allowed to evolve in a chemostat have an increased ability to attach to surfaces (Cox \& Gibson, 1974), we ran two control and two experimental chemostats with mixed populations of GR101 (an evolved derivative of P151) and P127. The results of one control and one experimental chemostat, representative for this set, are presented in Fig. 2.

GR101 was adapted to the chemostat environment and thus it increased in frequency in the liquid populations in both the control and the experimental cultures without the initial 25 generation lag seen in the P151 + P127 chemostats. The coexistence in the control chemostat, 


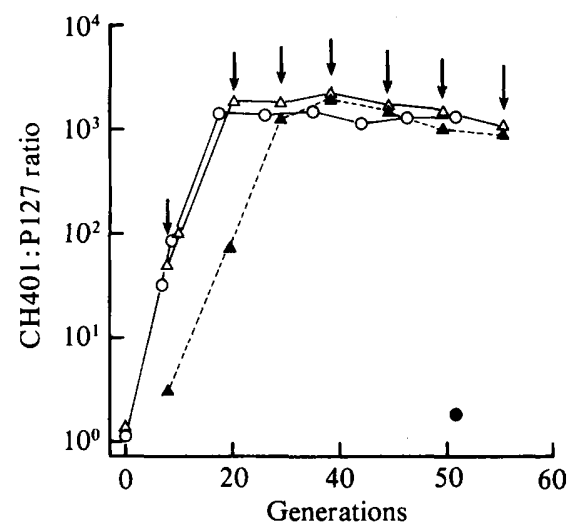

Fig. 3. Chemostats with mixed populations of $\mathrm{CH} 401$ (evolved, lac::Tn10) and P127 (mut Lac $^{+}$). Arrows denote times when the vessel of the experimental chemostat was changed. $O$, Liquid samples from the control chemostat;, wall samples from the control chemostat; $\Delta$, liquid samples from the experimental chemostat; $\Delta$, wall samples from the experimental chemostat.

however, was again dependent on a wall effect; GR101 and P127 coexisted in the liquid phase of the control chemostat, but not in the experimental culture. As in the control chemostat with P151 and P127 (Fig. 1), the final wall ratio and the final liquid ratio in the control GR101 + P127 chemostat at 206 generations $\left(1.68 \times 10^{1}\right.$ and $6.75 \times 10^{2}$, respectively) were markedly different.

The increased ability of GR101 to adhere to the vessel was demonstrated by the fact that in the experimental chemostat, at any one time, the GR101:P127 wall ratios were usually greater than the liquid ratios (Fig. 2). In the experimental chemostat with P151 and P127, the wall ratios had always been lower than the liquid ratios measured at the same time (Fig. 1). Nonetheless, the adhesive properties of GR101 did not eliminate the coexistence mediated by the wall populations of P127 in the control chemostat. GR101 colonized an unoccupied chemostat wall faster than P127, but it could not displace P127 bacteria that had already attached themselves to the vessel. As shown by Munson \& Bridges (1964), the ability of a strain to displace a second one from the chemostat wall will cause the elimination of the latter. In the present study, the permanency of the P127 wall population ensured the coexistence of the two strains in the chemostat.

\section{Chemostats with strains $\mathrm{CH} 401$ and P127}

To extend the study we examined the liquid phase coexistence between $\mathrm{CH} 401$, which is a mutator strain carrying the transposon $\mathrm{Tn} 10$, and P127. Like $m u t T 1, \mathrm{Tn} 10$ confers an advantage in chemostat cultures through mutagenesis (Chao et al., 1983). The results of a control and an experimental chemostat with mixed populations of $\mathrm{CH} 401$ and $\mathrm{P} 127$ are presented in Fig. 3 . CH401, like GR101, is an evolved strain and, consequently, the CH401: $\mathrm{P} 127$ liquid ratios increased immediately at the start of the culture. The liquid ratios in both the control and experimental chemostats, however, levelled out at about $10^{3}$. Thus, unlike GR101, CH401 coexisted with $\mathrm{P} 127$ in the liquid phase of the chemostat through a mechanism other than a wall effect. The difference between GR101 and $\mathrm{CH} 401$ was also accentuated by the fact that an an one time the $\mathrm{CH} 401$ : $\mathrm{P} 127$ wall ratio in the experimental chemostat was lower than the liquid ratio in the same culture. In this respect the behaviour of the $\mathrm{CH} 401$ wall population was more like that of the $\mathrm{P} 151$ wall population, suggesting that $\mathrm{CH} 401$ had not evolved an increased adhesive ability. Since our original intention was only to examine the effects of wall populations we did not further investigate the mechanism(s) allowing for the coexistence between $\mathrm{CH} 401$ and P127. This result with $\mathrm{CH} 401$ and P127 was replicated with an additional pair of control and experimental chemostats.

We were concerned at the start of this study that replacing the chemostat vessel could perturb a coexistence mediated by a mechanism other than a wall population and give the false impression that a wall effect was present. The results presented in Fig. 3 show so little difference 
between the control and experimental chemostats that the effects of replacing the vessel must be minimal. At least by our procedures the vessel replacement test can distinguish between a coexistence caused by a wall effect and one caused by an alternative mechanism.

In designing the experiments for this study we used the coexistence ratios in the control chemostats to determine how frequently to replace the vessels of the experimental chemostats. The coexistence ratios were $10^{2}$ to $10^{3}$ times greater than the starting ratios of the control chemostats. To ensure that we would observe the dynamics of the liquid populations without interference of a wall effect, we replaced the vessels every time the liquid ratio of strains in the experimental chemostats changed by a factor of about 10 . Since changes in the ratios of populations undergoing selection in a chemostat are usually log-linear (Moser, 1957; Powell, 1958; Chao et al., 1977; Hartl \& Dykhuizen, 1979), a reasonable guideline is to replace the vessel whenever the change is equal to the square root (the geometric half point) of the expected change from the start of the chemostat to the coexistence. A preliminary control chemostat is helpful for gauging the magnitude of the expected change.

We were able to estimate the fitness difference between P127 and the two mut T strains, P151 and GR101, in the liquid phase of our chemostats from the trajectories of the populations in Figs 1 and 2 . The estimates were obtained by a least squares fit of the data to the relationship $\Delta \log _{e}$ (liquid ratio) $=S \cdot \Delta t$, where $S$ is the fitness difference and $t$ is time measured in generations (Cox \& Gibson, 1974). For the control chemostats, we used the data points before the coexistence phase, and for the P151 and P127 chemostats, we further restricted the estimate to points after the initial 25 generation lag, since the inclusion of points in either the lag or the coexistence phase might have led to an underestimate (Chao \& Cox, 1983). We estimated $S=$ $0 \cdot 10$ and $0 \cdot 15$ per generation, respectively, from the control and experimental chemostats in Fig. 1 and $S=0 \cdot 21$ and $0 \cdot 25$ per generation, respectively, from the control and experimental chemostats in Fig. 2. The values of $S$ were positive because P151 and GR101 were more fit than $P 127$. In both sets the estimate from the experimental chemostats was higher, suggesting that we still underestimated $S$ with the data from the control chemostats. The problems of estimating $S$ from the control chemostats were twofold. First, the determination of when the coexistence began was often ambiguous. Second, by confining the estimate only to points before the coexistence phase we limited our sample size. With the experimental chemostats, the coexistence was eliminated and we were able to follow changes in the liquid populations for over 8 orders of magnitude and substantially increase the number of sample points. An accurate estimate of the selective difference between two bacterial strains in the liquid phase of a chemostat is often desired because that parameter can be an important component of mathematical models for chemostats (Moser, 1957; Powell, 1958; Stewart \& Levin, 1973; Taylor \& Williams, 1975; Hsu et al., 1977; Levin \& Stewart, 1980). Our recommendation is that the most accurate estimate will be made from cultures such as our experimental chemostats where the effects of the wall populations have been eliminated by repeated replacements of the culture vessel.

As we indicated earlier, strains carrying either the mutator allele mutTl or the transposon Tn 10 gain an advantage in mixed chemostat cultures by generating beneficial mutations at a higher rate (Cox \& Gibson, 1974; Chao \& Cox, 1983; Chao et al., 1983). The two evolved strains used in this study, the mutT-generated GR101 and the Tn10-generated $\mathrm{CH} 401$, were isolated from chemostats supported with identical growth medium and yet the liquid phase coexistence between GR101 and P127 was determined by a wall effect and that between CH401 and P127 was not. Furthermore, only GR 101 evolved an increased ability to adhere to the chemostat wall. GR101 could have acquired additional adaptations since it was allowed to evolve for a longer period than $\mathrm{CH} 401$ (534 and 219 generations, respectively). On the other hand, given that the mutTI allele and the transposon $\mathrm{Tn} 10$ generate mutations by different mechanisms (transversions versus transpositions: Cox \& Gibson, 1974; Chao \& Cox, 1983; Chao et al., 1983), they could also have generated mutations with different modes of action.

It is not clear whether the increased adhesive ability of GR101 was adaptive. When we paired GR101 with P127 in a control chemostat the two strains coexisted. GR101 colonized an unoccupied chemostat faster than P127, but it did not destroy the coexistence because it could 
not displace an established P127 wall population. Our results provided no indication that the increased adhesive ability of GR101 was adaptive in our chemostats. The initial rates of increase of GR101 relative to P127 in the liquid phase of both the control and experimental chemostats were identical (Fig. 2). It made no difference whether the GR101 liquid population was given new vessel walls to colonize or whether the GR101 wall population was maintained or periodically removed. Perhaps the enhanced adhesion was a pleiotropic effect linked with genetic changes favoured by selection in chemostats.

We thank Barbara A. Liepe, Bruce R. Levin and D. Dykhuizen for helpful criticisms of this manuscript. This research was supported by grant GM31990 (L. C.) from the National Institute of General Medical Sciences and funds from Northwestern University.

\section{REFERENCES}

Bungay, H. R. \& Bungay, M. L. (1968). Microbial interactions in continuous culture. Advances in Applied Microbiology 10, 269-290.

Chao, L. \& Cox, E. C. (1983). Competition between high and low mutating strains of Escherichia coli. Evolution 37, 125-134.

Chao, L., Levin, B. R. \& Stewart, F. M. (1977). A complex community in a simple habitat: an experimental study with bacteria and phage. Ecology 58, 369-378.

Chao, L., Vargas, C., Spear, B. B. \& Cox, E. C. (1983). Transposable elements as mutator genes in evolution. Nature, London 303, 633-635.

Contols, D. E. (1959). Kinetics of bacterial growth: relationship between population density and specific growth rate of continuous cultures. Journal of General Microbiology 21, 40-50.

Cox, E. C. \& Gibson, T. C. (1974). Selection for high mutation rates in chemostats. Genetics 77, 169-184.

Dykhuizen, D. (1978). Selection for tryptophan auxotrophs of Escherichia coli in glucose-limited chemostats as a test of the energy conservation hypothesis of evolution. Evolution 32, 125-150.

Dykhuizen, D. \& HaRTL, D. (1983). Studies of selection using chemostats. Microbiological Reviews 47, 150-168.

Edlin, G., Lin, L. \& Kudrna, R. (1975). Lambda lysogens of $E$. coli reproduce more rapidly than nonlysogens. Nature, London 255, 735-737.

Foster, T. J., Davis, M. A., Roberts, D. E., TAKeshita, K. \& Kleckner, N. (1981). Genetic organization of transposon Tn10. Cell 23, 201-213.

Freter, R., Stauffer, E., Cleven, D., Holdeman, L. \& MOORE, W. (1983). Continuous-flow cultures as in vitro models of the ecology of large intestinal flora. Infection and Immunity 39, 666-675.

Godwin, D. \& Slater, J. H. (1979). The influence of the growth environment on the stability of a drug resistant plasmid in Escherichia coli K 12. Journal of General Microbiology 111, 201-210.

HaRTL, D. \& Dykhuizen, D. (1979). A selectively driven molecular clock. Nature, London 281, $230-$ 231.

Hsu, S. B., Hubbell, S. \& Waltman, P. (1977). A mathematical theory for single-nutrient competition in continuous cultures of micro-organisms. SIAM Journal of Applied Mathematics 32, 366-383.

JanNaSCH, H. W. \& Mateles, R. I. (1974). Experimental bacterial ecology studied in continuous culture. Advances in Microbial Physiology 11, 165212.

KuBITSCHEK, H. E. (1970). Research with Continuous Cultures. New Jersey: Prentice-Hall.

LARSEN, D. H. \& Dimmick, R. L. (1964). Attachment and growth of bacteria on surfaces of continuousculture devices. Journal of Bacteriology 88, 1380 1387.

LEVIN, B. R. \& STEWART, F. M. (1980). The population biology of bacterial plasmids: a priori conditions for the existence of mobilizable nonconjugative factors. Genetics 87, 209-228.

MEERS, J. L. (1973). Growth of bacteria in mixed cultures. CRC Critical Reviews in Microbiology 2, 139-184.

MeERS, J. L. \& Tempest, D. W. (1968). The influence of extracellular products on the behaviour of mixed microbial populations in magnesium-limited chemostat cultures. Journal of General Microbiology 52, 309317.

Megee, R. D. III, Drake, J. F., Fredrickson, A. G. \& TsuchiYA, H. M. (1972). Studies in intermicrobial symbiosis. Saccharomyces cerevisiae and Lactobacillus casei. Canadian Journal of Microbiology 18, 17331742.

Moser, H. (1957). The dynamics of bacterial populations maintained in the chemostat. Publication, Carnegie Institute of Washington no. 614.

Munson, R. J. \& BRIDGES, B. A. (1964). 'Take-over' an unusual selection process in steady-state cultures of Escherichia coli. Journal of General Microbiology 37, 411-418.

Powell, E. O. (1958). Criteria for the growth of contaminants and mutants in continuous culture. Journal of General Microbiology 18, 259-268.

Shindala, A., Bungay, H. R., KRIEG, N. R. \& CULBERT, K. (1965). Mixed-culture interactions. Journal of Bacteriology 89, 693-696.

Stewart, F. M. \& Levin, B. R. (1973). Partitioning of resources and outcome of interspecific competition: a model and some general considerations. American Naturalist 107, 171-198.

Taylor, P. A. \& Williams, P. J. LeB. (1975). Theoretical studies on the coexistence of competing species under continuous-flow conditions. Canadian Journal of Microbiology 21, 90-98.

TEMPEST, D. W. (1970). The continuous cultivation of micro-organisms. I. Theory of the chemostat. Methods in Microbiology 2, 260-276. 
VeilleUX, B. G. \& RowLAND, I. (1981). Simulation of the rat intestinal ecosystem using a two-stage continuous culture system. Journal of General Microbiology 123, 102-115.

VOGEL, H. J. \& BONNER, D. M. (1956). Acetylornithinase of Escherichia coli: partial purification and some properties. Journal of Biological Chemistry 218, 97106.

ZAMENHOF, S. \& EICHHORN, H. H. (1967). Study of microbial evolution through loss of biosynthetic functions: establishment of "defective" mutants. Nature, London 216, 455-458 